\title{
NEIGHBORHOOD SPACES
}

\section{C. KENT and WON KEUN MIN}

Received 7 February 2002

\begin{abstract}
Neighborhood spaces, pretopological spaces, and closure spaces are topological space generalizations which can be characterized by means of their associated interior (or closure) operators. The category NBD of neighborhood spaces and continuous maps contains PRTOP as a bicoreflective subcategory and CLS as a bireflective subcategory, whereas TOP is bireflectively embedded in PRTOP and bicoreflectively embedded in CLS. Initial and final structures are described in these categories, and it is shown that the Tychonov theorem holds in all of them. In order to describe a successful convergence theory in NBD, it is necessary to replace filters by more general $p$-stacks.
\end{abstract}

2000 Mathematics Subject Classification: 54A05, 54A10.

1. Introduction. In his original definition of topology, Hausdorff [7] assigned to each point $x$ in a set $X$ a system of neighborhoods subject to certain axioms. We will define a neighborhood structure $v$ on $X$ in the same way, using axioms weaker than those of Hausdorff, but just strong enough to include both pretopological and closure spaces as special cases of neighborhood spaces. Of particular significance is the fact that pretopologies, supratopologies, and neighborhood structures on $X$ are all uniquely determined by their associated interior (or closure) operators, which, in each case, can be axiomatically described by generalizing in different ways the Kuratowski interior operator that characterizes a topology.

To better understand the preceding statements, we recall that a topological space $(X, \tau)$ has an interior operator $I$ which satisfies the following Kuratowski interior axioms:

(i) $I(X)=X$;

(ii) $I(A) \subseteq A$, for all $A \subseteq X$;

(iii) $I(A \cap B)=I(A) \cap I(B)$, for all $A, B \in 2^{X}$;

(iv) $I(I(A))=I(A)$, for all $A \subseteq X$.

Furthermore, if $I: 2^{X} \rightarrow 2^{X}$ is any set function satisfying these axioms, then $I$ determines a unique topology on $X$ whose interior operator is $I$.

Pretopological spaces were introduced by Choquet [4] in 1948. A pretopology $p$ on $X$ is defined by assigning at each $x \in X$ a filter of neighborhoods which, unlike a topology, is not required to have a filter base of open sets. The interior operator $I$ determined by a pretopology satisfies axioms (i), (ii), and (iii) and conversely any set function $I$ satisfying these three axioms determines a unique pretopology on $X$ whose associated interior operator is $I$. Pretopological spaces can also be characterized using the three corresponding closure axioms, and, indeed, they are studied under the name closure spaces by Čech in [3]. 
During the past forty years, there has been a growing interest in what Njästad [10] calls nearly open sets, which include semi-open sets $[8,10]$, as well as preopen and semi-preopen sets [1]. A supratopology, [9], is defined to be a collection of subsets of a set $X$ (called supraopen sets) which contains $X$ and is closed under arbitrary unions, but, unlike a topology, is not required to be closed under finite intersections. If $(X, \tau)$ is a topological space, the collections of semi-open, preopen, and semi-preopen sets relative to $\tau$ each form supratopologies on $X$ derived from $\tau$. Given a supratopology $\sigma$ on $X$, the interior of any set is its largest supraopen subset, and the interior operator thus obtained satisfies axioms (i), (ii), and (iv), along with an additional axiom: (v) if $A \subseteq B$, then $I(A) \subseteq I(B)$ which is, in fact, a generalization of (iii).

Furthermore, if $I: 2^{X} \rightarrow 2^{X}$ is any set function satisfying (i), (ii), (iv), and (v), then the collection $\{A \subseteq X: I(A)=A\}$ is a supratopology on $X$ whose associated interior operator is $I$.

A pair $(X, \sigma)$ consisting of a set $X$ with a supratopology $\sigma$ will be called a closure space $[5,6]$. Closure spaces are typically characterized by the closure axioms corresponding to axioms (i), (ii), (iv), and (v) of the preceding paragraph. Their study is partially motivated by Birkhoff's study of the relation between closures and certain Galois connections [2]. A brief discussion of motivations and applications for closure spaces is found in [5]; these are quite independent of those mentioned in the preceding paragraph, although the resulting structures are equivalent.

Throughout this paper, we use the term interior operator on $X$ to mean a set function $I: 2^{X} \rightarrow 2^{X}$ which satisfies (i), (ii), and (v); the interior operators for topological, pretopological, and closure spaces are special cases. Given any interior operator $I$ on $X$ and $x \in X$, a subset $V$ is called a neighborhood of $x$ if $x \in I(V)$. In this way, each interior operator $I$ determines a collection of subsets of $X$ for each $x \in X$, and these neighborhood collections, when appropriately axiomatized in Section 2, will define a neighborhood structure $v$ on $X$, and the pair $(X, v)$ is called a neighborhood space. The natural morphisms between neighborhood spaces are called continuous maps.

The category NBD of neighborhood spaces and continuous maps contains, as full subcategories, the category PRTOP of pretopological spaces, CLS of closure spaces, and TOP of topological spaces. By studying initial and final structures in these categories, we show that PRTOP is bicoreflectively embedded in NBD, CLS is bireflectively embedded in NBD, and TOP is bireflectively embedded in PRTOP and bicoreflectively embedded in CLS. Some properties appropriate to the category NBD, including compactness, are investigated in Section 5, where it is shown that the Tychonov theorem holds in all of the categories cited above. It is also shown that a satisfactory convergence theory can be obtained for neighborhood and closure spaces if filter is replaced by the more general notion of a $p$-stack. A new notion of " $p$-compactness" based on ultra $p$-stacks is introduced and shown to be preserved under arbitrary products in NBD and CLS.

2. Neighborhood spaces. In order to develop the theory of neighborhood spaces, it is necessary to introduce a new vehicle for describing convergence. Given a set $X$, a collection $\mathscr{C}$ of subsets of $X$ is called a stack if $A \in \mathscr{C}$ whenever $B \in \mathscr{C}$ and $B \subseteq A$; a stack which is closed under finite intersections and does not contain the empty set is 
called a filter. The concept we need is intermediate in generality between a stack and a filter.

DEFINITION 2.1. A stack $\mathscr{H}$ on a set $X$ is called a $p$-stack if it satisfies the following condition:

(p) $A, B \in \mathscr{H}$ implies $A \cap B \neq \varnothing$.

Condition (p) is called the pairwise intersection property (PIP) which is strictly weaker than the well-known finite intersection property (FIP). A collection $\mathscr{B}$ of subsets of $X$ with the PIP is called a $p$-stack base. For any collection $\mathscr{B}$, we denote by $\langle\mathscr{B}\rangle=\{A \subseteq X$ : $\exists B \in \mathscr{B}$ such that $B \subseteq A$ \} the stack generated by $\mathscr{B}$, and if $\mathscr{B}$ is $p$-stack base, then $\langle\mathscr{B}\rangle$ is a $p$-stack. If $\mathscr{B}$ is a $p$-stack base with the FIP, then $\mathscr{B}$ is a filter subbase, and in this case $\mathscr{B}$ generates the filter $[\mathscr{B}]=\left\{A \subseteq X: \exists B_{1}, \ldots, B_{n} \in \mathscr{B}\right.$ such that $\left.\bigcap_{i=1}^{n} B_{i} \subseteq A\right\}$.

Let $\mathrm{pS}(X)$ (resp., $\mathbb{F}(X)$ ) denote the set of all $p$-stacks (resp., filters) on $X$, partially ordered by inclusion. The maximal elements in $\mathrm{pS}(X)$ (resp., $\mathbb{F}(X)$ ) are called ultra $p$ stacks (resp., ultrafilters). We may easily verify that every ultrafilter is an ultrapstack, and (via Zorn's lemma) that every $p$-stack is contained in an ultrapstack.

LEMMA 2.2. For $\mathscr{H} \in \mathrm{pS}(X)$, the following are equivalent:

(a) $\mathcal{H}$ is an ultrapstack;

(b) if $A \cap H \neq \varnothing$, for all $H \in \mathscr{H}$, then $A \in \mathscr{H}$;

(c) $B \notin \mathscr{H}$ implies $X \backslash B \in \mathscr{H}$.

Proof. (a) $\Rightarrow$ (b). If $A \cap H \neq \varnothing$, for all $H \in \mathscr{H}$, and $A \notin \mathscr{H}$, then $\mathscr{H} \cup\langle A\rangle$ would be a $p$-stack strictly larger than $\mathscr{H}$.

(b) $\Rightarrow$ (c). If $B \notin \mathscr{H}$, then, by (b), there is $H \in \mathscr{H}$ such that $B \cap H=\varnothing$. Thus $H \subseteq X \backslash B$, which implies $X \backslash B \in \mathscr{H}$.

(c) $\Rightarrow$ (a). Let $\mathscr{H} \subseteq \mathscr{K}$, where $\mathscr{K} \in \mathrm{pS}(X)$ and $K \in \mathscr{K}$. If $K \notin \mathscr{H}$, then by (c) $X \backslash K \in \mathscr{H}$, and hence $X \backslash K \in \mathscr{K}$, a contradiction.

If $X$ contains at least three distinct elements $a, b, c$, the $p$-stack base $\{\{a, b\},\{a, c\}$, $\{b, c\}\}$ generates an ultrapstack which is not an ultrafilter. If $A \subseteq X$ is nonempty, $\langle A\rangle$ denotes the filter of all oversets of $A$, and $\dot{x}=\langle\{x\}\rangle$ denotes the ultrafilter generated by $\{x\}$.

For a function $f: X \rightarrow Y$ and $\mathscr{H} \in \operatorname{pS}(X)$, the image stack $f(\mathscr{H})$ in $\operatorname{pS}(Y)$ has $p$ stack base $\{f(H): H \in \mathscr{H}\}$. Likewise, if $\mathscr{G} \in \mathrm{pS}(Y), f^{-1}(\mathscr{G})$ denotes the $p$-stack on $X$ generated by $\left\{f^{-1}(G): G \in \mathscr{G}\right\}$.

Proposition 2.3. Let $f: X \rightarrow Y$ be a function, $\mathscr{H} \in \operatorname{pS}(X)$. Then

(a) if $\mathscr{H}$ is a filter, so is $f(\mathscr{H})$;

(b) if $\mathscr{H}$ is an ultrafilter, so is $f(\mathscr{H})$;

(c) if $\mathscr{H}$ is an ultrapstack, so is $f(\mathcal{H})$.

Proof. (c) If $B \cap f(H) \neq \varnothing$, for all $H \in \mathscr{H}$, then $f^{-1}(B) \cap H \neq \varnothing$, for all $H \in \mathscr{H}$, and by Lemma 2.2, $f^{-1}(B) \in \mathscr{H}$, which implies $B \in f(\mathscr{H})$, and thus $f(\mathscr{H})$ is an ultrapstack.

In 1915, Hausdorff gave the first definition of what was later called a topology by assigning to each point $x$ in a set $X$ a family of neighborhoods subject to certain axioms. We use the same approach for defining a neighborhood structure. 
Definition 2.4. Let $X$ be a set, and let $v \subseteq \mathrm{pS}(X)$ be given by $v=\{v(x): x \in X\}$, where $v(x) \subseteq \dot{x}$, for all $x \in X$. Then $v$ is called a neighborhood structure on $X, v(x)$ is called the $\nu$-neighborhood stack at $x$, and $(X, v)$ is called a neighborhood space. For convenience, neighborhood will be henceforth abbreviated by nbd.

Let $\mathrm{N}(X)$ be the set of all nbd structures on $X$, partially ordered as follows: $\nu \leq \mu$ if and only if $\nu(x) \leq \mu(x)$, for all $x \in X$ (in which case $\nu$ is coarser than $\mu$ and $\mu$ is finer than $v$ ). A $p$-stack $\mathscr{H}$ on $X v$-converges to $x$ (written $\mathscr{H} \stackrel{v}{\rightarrow} x$ ) if $v(x) \subseteq \mathscr{H}$.

DEFINITION 2.5. If $(X, v)$ and $(Y, \mu)$ are nbd spaces, then $f:(X, v) \rightarrow(Y, \mu)$ is continuous if $\mu(f(x)) \subseteq f(\nu(x))$, for all $x \in X$, and $f$ is interior if $f(\nu(x)) \subseteq \mu(f(x))$, for all $x \in X$. A continuous, interior bijection is called a homeomorphism, and in this case $(X, \nu)$ and $(Y, \mu)$ are homeomorphic.

Proposition 2.6. The function $f:(X, v) \rightarrow(Y, \mu)$ is continuous if and only if, whenever $\mathscr{H} \in \mathrm{pS}(X)$ and $\mathscr{H} \stackrel{v}{\rightarrow} x, f(\mathscr{H}) \stackrel{\mu}{\rightarrow} f(x)$.

The category having neighborhood spaces as objects and continuous maps as morphisms is denoted by NBD.

If $(X, v)$ is an nbd space and $A \subseteq X$, let

$$
\begin{aligned}
I_{v}(A) & =\{x \in A: A \in v(x)\} \\
\mathrm{Cl}_{\nu}(A) & =\{x \in X: A \cap V \neq \varnothing, \forall V \in \mathcal{v}(x)\} .
\end{aligned}
$$

Proposition 2.7. If $(X, v)$ is a neighborhood space, $A \subseteq X$, then

(a) $I_{v}$ satisfies axiom (i), (ii), (v);

(b) $I_{v}(A)=\{x \in A: A \in \mathscr{H}$, for every $p$-stack $\mathscr{H} \stackrel{v}{\rightarrow} x\}$;

(c) $\mathrm{Cl}_{v}(A)=X \backslash I_{v}(X \backslash A)$;

(d) $\mathrm{Cl}_{v}(A)=\{x \in X: \exists \mathscr{H} \in \mathrm{pS}(X)$ such that $\mathscr{H} \stackrel{v}{\rightarrow} x$ and $A \in \mathscr{H}\}$.

Proof. (d) If $x \in \mathrm{Cl}_{v}(A)$, let $\mathscr{H}=v(x) \cup\langle A\rangle$. If $x \notin \mathrm{Cl}_{v}(A)$, some $V \in v(x)$ has an empty intersection with $A$, and so no $p$-stack containing $A$ can $v$-converge to $x$.

THEOREM 2.8. Let $\square(X)$ be the set of all interior operators on $X$, partially ordered by $I_{1} \leq I_{2}$ if and only if $I_{1}(A) \subseteq I_{2}(A)$, for all $A \subseteq X$. If $\varphi: \mathrm{N}(X) \rightarrow \square(X)$ is defined by $\varphi(v)=I_{v}$, then $\varphi$ is an order isomorphism.

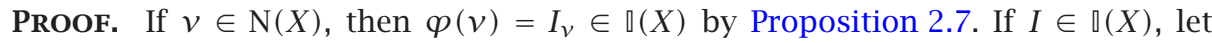
$\psi(I)=v_{I}$, where $v_{I}(x)=\{A \subseteq X: x \in I(A)\}$, for each $x \in X$. Then $\psi: \mathbb{\square}(X) \rightarrow \mathrm{N}(X)$ follows easily. To show $I_{v_{I}}=I$, note that for all $A \subseteq X, x \in I(A)$ if and only if $A \in v_{I}(x)$ if and only if $x \in I_{v}(A)$ if and only if $A \in \nu_{I_{v}}(x)$. Thus $\varphi \circ \psi$ is the identity map on $\square(X)$ and $\psi \circ \varphi$ is the identity map on $\mathrm{N}(X)$, where $\varphi$ is a bijection. Finally, $\nu \leq \mu$ in $\mathrm{N}(X)$ if and only if $\nu(x) \subseteq \mu(x)$, for all $x \in X$ if and only if $A \in \nu(x)$ implies $A \in \mu(x)$ if and only if $I_{v}(A) \subseteq I_{\mu}(A)$, for all $A \subseteq X$ if and only if $\varphi(\nu) \leq \varphi(\mu)$.

Proposition 2.9. For a function $f:(X, v) \rightarrow(Y, \mu)$ between neighborhood spaces, (1) if and only if (2) if and only if (3) and (4) if and only if (5) if and only if (6). 
(1) $f$ is continuous;

(2) $f^{-1}\left(I_{\mu}(A)\right) \subseteq I_{\nu}\left(f^{-1}(A)\right)$, for all $A \subseteq Y$;

(3) $f\left(\mathrm{Cl}_{v}(B)\right) \subseteq \mathrm{Cl}_{\mu} f(B)$, for all $B \subseteq X$;

(4) $f$ is interior;

(5) $I_{\nu}\left(f^{-1}(A)\right) \subseteq f^{-1}\left(I_{\mu}(A)\right)$, for all $A \subseteq Y$;

(6) $f^{-1}\left(\mathrm{Cl}_{\mu}(B)\right) \subseteq \mathrm{Cl}_{v} f^{-1}(B)$, for all $B \subseteq Y$.

If $(X, v)$ is a neighborhood space and $\mathscr{H} \in \mathrm{pS}(X)$, the associated closure $p$-stack $\mathrm{Cl}_{v}(\mathscr{H})$ and neighborhood $p$-stack $\mathscr{V}_{v}(\mathscr{H})$ are generated by the $p$-stack bases $\left\{\mathrm{Cl}_{v} H\right.$ : $H \in \mathscr{H}\}$ and $\left\{A \subseteq X: I_{v} A \in \mathscr{H}\right\}$, respectively. Note that $\mathscr{V}_{v}(\dot{x})=v(x)$ is the $v$-nbd stack at $x$.

Proposition 2.10. The interior (or closure) operator for an $n b d$ space $(X, v)$ is idempotent if and only if $\mathscr{V}_{v}(v(x))=v(x)$, for all $x \in X$.

Proof. Let $\mathscr{V}_{v}(v(x))=v(x)$, for all $x \in X$. If $A \subseteq X$ and $x \in I_{v}(A)$, then $A \in$ $\mathscr{V}_{v}(v(x))$ implies $I_{v}(A) \in v(x)$, and so $x \in I_{v}\left(I_{v}(A)\right)$. Thus $I_{v}=I_{v} \cdot I_{v}$. The converse is obvious.

DEFINITION 2.11. A neighborhood space $(X, v)$ is defined to be

(a) pretopological if $v(x)$ is a filter, for all $x \in X$;

(b) regular if $\mathrm{Cl}_{v}(v(x))=v(x)$, for all $x \in X$;

(c) supratopological if $\mathscr{V}_{v}(v(x))=v(x)$, for all $x \in X$;

(d) topological if pretopological and supratopological.

The terms pretopology, supratopology, and topology will be used for an nbd structure $v$ which is pretopological, supratopological, or topological. Also, a pretopological nbd space $(X, v)$ will be called a pretopological space; likewise for topological space. A supratopological nbd space will be called a closure space.

THEOREM 2.12. A neighborhood space $(X, v)$ is

(a) pretopological if and only if $I_{v}$ satisfies (i), (ii), and (iii);

(b) supratopological if and only if $I_{v}$ satisfies (i), (ii), (iv), and (v);

(c) topological if and only if $I_{v}$ satisfies (i), (ii), (iii), and (iv).

Proof. (c) is well known and (b) follows by Proposition 2.10.

(a) If $v$ is pretopological and $A, B$ are subsets of $X$, then $I_{v}(A \cap B) \subseteq I_{v}(A) \cap I_{v}(B)$ follows by (v). If $x \in I_{v}(A) \cap I_{v}(B)$, then $A, B \in v(x)$ implies $A \cap B \in v(x)$, since $v(x)$ is a filter, and hence $I_{v}(A) \cap I_{v}(B) \subseteq I_{v}(A \cap B)$. Conversely, if $A, B \in v(x)$, then $x \in$ $I_{v}(A) \cap I_{v}(B)=I_{v}(A \cap B)$, and hence $A \cap B \in v(x)$, so $v(x)$ is a filter.

Whereas pretopologies are well known, additional discussion of supratopologies is appropriate. In 1983, Mashhour et al. [9] defined a supratopology $\sigma$ on $X$ to be a subcollection of $2^{X}$ containing $X$ and closed under arbitrary unions. The members of $\sigma$ are called supraopen sets. For example, if $(X, \tau)$ is a topological space, the collections $\mathrm{SO}(X)=\left\{A \subseteq X: A \subseteq \mathrm{Cl}_{\tau} I_{\tau} A\right\}$ of semi-open sets, [8], and $\mathrm{PO}(X)=\{A \subseteq X: A \subseteq$ $\left.I_{\tau} \mathrm{Cl}_{\tau} A\right\}$ of preopen sets, [1], are supratopologies on $X$ derived from $\tau$. It is easy to verify that our Definition 2.11(c) is equivalent to that given in [9]. Indeed, if $(X, \sigma)$ is a supratopological space as defined in [9] and $v_{\sigma}$ is the nbd structure defined by 
$\nu_{\sigma}(x)=\langle\{U \in \sigma: x \in U\}\rangle$, then $\nu_{\sigma}$ has an idempotent interior operator and is thus a supratopological nbd space. On the other hand, if $(X, v)$ is a supratopological nbd space, then $\sigma_{v}=\left\{U \subseteq X: I_{v} U=U\right\}$ is a supratopology in the sense of [9], and $v_{\sigma_{v}}=v$. The remaining details are left to the reader. The term closure space is used rather than supratopological nbd space for brevity and consistency with current usage (see [5, 6]).

The full subcategories of NBD determined by the pretopological, regular, supratopological, and topological nbd spaces are denoted, respectively, by PRTOP, REGNBD, CLS, and TOP. In the next two sections we study initial and final properties in these categories to determine how the smaller categories are embedded in the larger ones.

3. The lattice $\mathrm{N}(X)$. For any nonempty set $X$, let $\mathrm{PR}(X), \mathrm{ST}(X), \mathrm{T}(X)$, and $\mathrm{RN}(X)$ denote the sets of pretopologies, supratopologies, topologies, and regular nbd structures on $X$, respectively, all partially ordered as subsets of $\mathrm{N}(X)$. In each case the resulting partially ordered set is a complete lattice, with the discrete and indiscrete topologies as greatest and least elements.

LEMMA 3.1. If $\mathscr{A}=\left\{v_{i}: i \in J\right\} \subseteq \mathrm{N}(X), v=\inf _{\mathrm{N}(X)} \mathscr{A}$, and $\mu=\sup _{\mathrm{N}(X)} \mathscr{A}$, then $v(x)=$ $\cap\left\{v_{i}(x): i \in J\right\}$ and $\mu(x)=\cup\left\{v_{i}(x): i \in J\right\}$.

Proposition 3.2. Let $\mathscr{A}=\left\{v_{i}: i \in J\right\} \subseteq \mathrm{N}(X)$. Then

(1) if each $v_{i} \in \mathscr{A}$ is a supratopology, so is $\sup _{\mathrm{N}(X)} \mathscr{A}$;

(2) if each $v_{i} \in \mathscr{A}$ is a regular, so is $\sup _{\mathrm{N}(X)} \mathscr{A}$;

(3) if each $v_{i} \in \mathscr{A}$ is a pretopology, so is $\inf _{\mathrm{N}(X)} \mathscr{A}$;

(4) if each $v_{i} \in \mathscr{A}$ is a topology, so are $\sup _{\mathrm{PR}(X)} \mathscr{A}$ and $\inf _{\mathrm{ST}(X)} \mathscr{A}$.

Proof. (1) Let $\mu=\sup _{\mathrm{N}(X)} \mathscr{A}$; to show $\mu$ is a supratopology, it suffices to show that $V \in \mu(x)$ implies $I_{\mu}(V) \in \mu(x)$. By Lemma 3.1, $V \in \mu(x)$ implies $V \in v_{j}(x)$ for some $\jmath \in J$, and since $v_{j}$ is a supratopology, $I_{v_{j}}(V) \in v_{j}(x)$. But $v_{J} \leq \mu$ implies $I_{v_{j}}(V) \subseteq$ $I_{\mu}(V)$, so $I_{\mu}(V) \in \mu(x)$.

(2) Let $\mu=\sup _{\mathrm{N}(X)} \mathscr{A}, x \in X$, and $V \in \mu(x)$. Then $V \in v_{\jmath}(x)$ for some $\jmath \in J$, and since $v_{j}$ is regular, there is $W \in \nu_{j}(x)$ such that $\mathrm{Cl}_{v_{J}} W \subseteq V$. Since $v_{j} \leq \mu, \mathrm{Cl}_{\mu} W \subseteq V$ and $W \in v_{j}(x)$ implies $W \in \mu(x)$. Thus $\mu$ is regular.

(3) Let $v=\inf _{\mathrm{N}(T)} \mathscr{A}$; to show $v$ is a pretopology, it suffices to show that each $v(x)$ is a filter for arbitrary $x \in X$. By Lemma 3.1, $v(x)=\bigcap_{i \in J} v_{i}(x)$, and $v(x)$ is a filter since each $v_{i}(x)$ is a filter.

(4) The first part is well known, and the second follows because any intersection of topologies on $X$ is again a topology.

If $A$ is a complete lattice and $B \subseteq A$ has the inherited order, we say that $B$ is supdense (resp., inf-dense) in $A$ if every $a \in A$ is the supremum (resp., infimum) in $A$ of members of $B$. Furthermore, $B$ is said to be order dense in $A$ if $A$ is the smallest sub-complete lattice of itself containing $B$.

THEOREM 3.3. (1) ST $(X)$ is inf-dense in $\mathrm{N}(X)$.

(2) $\mathrm{PR}(X)$ is sup-dense in $\mathrm{N}(X)$.

(3) $\mathrm{T}(X)$ is sup-dense in $\mathrm{ST}(X)$.

(4) $\mathrm{T}(X)$ is inf-dense in $\mathrm{PR}(X)$. 
Proof. (1) Let $v \in \mathrm{N}(X)$ and, for each $y \in X$, let $\mu_{y}$ be defined by

$$
\mu_{y}(x)= \begin{cases}v(y), & y=x \\ \dot{x}, & y \neq x\end{cases}
$$

We may easily verify that $\mu_{y} \in \mathrm{ST}(X)$ and $\nu=\inf _{\mathrm{N}(X)}\left\{\mu_{y}: y \in X\right\}$.

(2) Assume $x \in A \subseteq X$, and let $\mu_{A, x}$ be the pretopology defined by

$$
\mu_{A, x}(y)= \begin{cases}{[A],} & y=x \\ \{X\}, & y \neq x\end{cases}
$$

Then $v(x)=\bigcup\left\{\mu_{A, x}(x): A \in v(x)\right\}$, and by Lemma 3.1, $v=\sup _{\mathrm{N}(X)}\left\{\mu_{A, x}: A \in v(x)\right.$, $x \in X\}$.

(3) If $\sigma$ is a supratopology expressed as a collection of supraopen sets and $U \in \sigma$, let $\boldsymbol{\tau}_{U}$ be the topology $\{\varnothing, U, X\}$. Obviously $\sigma=\sup _{\mathrm{N}(X)}\left\{\boldsymbol{\tau}_{U}: U \in \sigma\right\}$.

(4) If $v$ is a pretopology, then $\mu_{y}$ (as defined in the proof of (1)) is a topology, and $v=\inf _{\mathrm{N}(X)}\left\{\mu_{y}: y \in X\right\}$.

COROLlary 3.4. $\mathrm{PR}(X), \mathrm{ST}(X)$, and $\mathrm{T}(X)$ are order dense in $\mathrm{N}(X)$.

Proposition 3.5. Let $(X, v)$ be an nbd space. There is a finest supratopology $\sigma v$ coarser than $v$, a finest regular nbd structure $\rho v$ coarser than $v$, and a coarsest pretopology $\pi v$ finer than $v$.

Proof. Since the set $S(v)$ of all supratopologies on $X$ coarser than $v$ is nonempty (it contains the indiscrete topology), $\sigma v=\sup _{\mathrm{N}(X)} S(v)$. Similarly, the existence of $\rho v$ and $\pi v$ is obtained with the help of Proposition 3.2.

The structures $\sigma v, \rho v$, and $\pi v$ are called, respectively, the supratopological, regular, and pretopological modifications of $\nu$. In the case of $\sigma v$ and $\pi v$, there are simple characterizations:

$$
\begin{aligned}
\sigma v & =\left\{U \subseteq X: I_{v} U=U\right\} \\
\pi v(x) & =[v(x)], \quad \forall x \in X .
\end{aligned}
$$

THEOREM 3.6. (1) If $v$ is a pretopology, $\sigma v$ is a topology.

(2) If $v$ is a supratopology, $\pi v$ is a topology.

Proof. (1) Let $U, V \in \sigma v$. Since $v$ is a pretopology, $I_{v}(U \cap V)=I_{v}(U) \cap I_{v}(V)=$ $U \cap V$. Thus $U \cap V \in \sigma v$, so $\sigma v$ is a topology.

(2) It suffices to show that $I_{\pi v}$ is idempotent. Let $A \subseteq X$ and $x \in I_{\pi v} A$. Since $v$ is a supratopology, there are $V_{1}, \ldots, V_{n}$ in $v(x)$ such that $I_{v} V_{j}=V_{\jmath}, j=1, \ldots, n$, and $\bigcap_{i=1}^{n} V_{i} \subseteq A$. Since $\pi v$ is a pretopology and $v \leq \pi v, x \in \bigcap_{i=1}^{n} I_{v}\left(I_{v}\left(V_{i}\right)\right) \subseteq \bigcap_{i=1}^{n} I_{\pi v}\left(V_{i}\right)$ $=I_{\pi v}\left(I_{\pi v}\left(\bigcap_{i=1}^{n} V_{i}\right)\right) \subseteq I_{\pi v} I_{\pi v}(A)$.

COROLLARY 3.7. (1) If $(X, v)$ is a pretopological space, then the topological modification $\sigma v$ is the finest topology on $X$ coarser than $v$. 
(2) If $(X, v)$ is a closure space, then the topological modification $\pi v$ is the coarsest topology on $X$ finer than $v$.

The terminology of Corollary 3.7 is not ambiguous since the only structures which are both pretopological and supratopological are topologies, for which the topological modification is the structure itself.

4. Initial and final structures. Let $X$ be a set, $\left\{\left(X_{i}, v_{i}\right): i \in J\right\}$ a collection of nbd spaces, $\left\{f_{i}: i \in J\right\}$ a corresponding collection of functions $f_{i}: X \rightarrow X_{i}$. If there is a coarsest nbd structure $v$ on $X$, making each $f_{i}:(X, v) \rightarrow\left(X_{i}, v_{i}\right)$ continuous, then $v$ is called the initial nbd structure on $X$ induced by the given collections of spaces and functions. Initial structures in the categories PRTOP and CLS are defined analogously. Final structure is the dual notion obtained by reversing all arrows and replacing coarsest by finest. An initial property $\mathrm{P}$ is one preserved under formation of initial structures (i.e., if each $\left(X_{i}, v_{i}\right)$ has property $\mathrm{P}$, so does $\left.(X, v)\right)$; final property is defined dually.

Proposition 4.1. Let $X$ be a set, $\left\{\left(X_{i}, v_{i}\right): i \in J\right\}$ a collection of nbd spaces, $\left\{f_{i}\right.$ : $i \in J\}$ a collection of functions $f_{i}: X \rightarrow X_{i}$. The initial structure $v$ on $X$ exists and is specified by $v(x)=\bigcup_{i \in J} f_{i}^{-1}\left(v_{i}\left(f_{i}(x)\right)\right)$, for all $x \in X$.

PRoof. For each $j \in J$, the nbd structure $\mu_{j}$ on $X$ defined by $\mu_{y}(x)=f_{j}^{-1}\left(v_{j}\left(f_{j}(x)\right)\right)$ is the coarsest making $f_{j}:\left(X, \mu_{j}\right) \rightarrow\left(X_{j}, \nu_{j}\right)$ continuous. Thus, $v=\sup _{\mathrm{N}(X)}\left\{\mu_{i}: i \in J\right\}$ is the coarsest nbd structure making $f_{i}:(X, v) \rightarrow\left(X_{i}, v_{i}\right)$ continuous for all $i \in J$, and the asserted characterization for $v(x)$, for any $x \in X$, follows by Lemma 3.1.

Proposition 4.2. Let $v$ be the initial nbd structure on $X$ induced by $\left\{\left(X_{i}, v_{i}\right): i \in J\right\}$ and $\left\{f_{i}: i \in J\right\}$, where $f_{i}: X \rightarrow X_{i}$. Let $\mathscr{H}$ be a $p$-stack on $X$ and $x \in X$. Then $\mathscr{H} \stackrel{v}{\rightarrow} x$ if and only if $f_{i}(\mathscr{H}) \stackrel{v_{i}}{\rightarrow} f_{i}(x)$, for all $i \in J$.

Proof. If $f_{i}(\mathscr{H}) \stackrel{v_{i}}{\longrightarrow} f_{i}(x)$, for all $i \in J$, then $v_{i}\left(f_{i}\left(x_{i}\right)\right) \subseteq f_{i}(\mathscr{H})$, and thus $f_{i}^{-1}\left(\nu_{i}\left(f_{i}(x)\right)\right) \subseteq f_{i}^{-1}\left(f_{i}(\mathscr{H})\right)$, for all $i \in J$. Therefore, $v(x) \subseteq \bigcup_{i \in J} f_{i}^{-1}\left(f_{i}(\mathscr{H})\right)$ follows by Proposition 4.1. If $A \in v(x)$, then $A \in f_{j}^{-1}\left(f_{j}(\mathscr{H})\right)$ for some $j \in J$. Consequently, there is $H \in \mathscr{H}$ such that $f_{j}^{-1}\left(f_{j}(H)\right) \subseteq A$, implying $H \subseteq A$, and hence $A \in \mathscr{H}$. Thus $v(x) \subseteq \mathscr{H}$ and $\mathscr{H} \stackrel{v}{\rightarrow} x$.

Proposition 4.3. Let $X$ be a set, $\left\{\left(X_{i}, v_{i}\right): i \in J\right\}$ a collection of nbd spaces, $\left\{f_{i}: i \in J\right\}$ a corresponding collection of functions $f: X_{i} \rightarrow X$. The final structure $\mu$ on $X$ exists and is specified by

(1) if $x \in \bigcup_{i \in J} f_{i}\left(X_{i}\right), \mu(x)=\left\{A \subseteq X: f_{i}^{-1}(A) \in v_{i}(a)\right.$, for all $a \in f_{i}^{-1}(x)$ and for all $i \in J$ such that $\left.x \in f_{i}\left(X_{i}\right)\right\}$;

(2) otherwise, $\mu(x)=\dot{x}$.

Proof. Clearly, $\mu \in \mathrm{N}(X)$. If $f_{i}:\left(X_{i}, v_{i}\right) \rightarrow(X, \mu)$ and $a \in X_{i}$, then $A \in \mu\left(f_{i}(a)\right)$, so $f_{i}^{-1}(A) \in v_{i}(a)$; thus $\mu\left(f_{i}(a)\right) \subseteq f_{i}\left(v_{i}(a)\right)$, which implies that $f_{i}$ is continuous. It is routine to verify that $\mu$ as specified is the finest nbd structure on $X$, making each $f_{i}:\left(X_{i}, v_{i}\right) \rightarrow(X, \mu)$ continuous.

THEOREM 4.4. (1) Regular and supratopological are initial properties in NBD.

(2) Pretopological is a final property in NBD. 
Proof. In the notation and terminology of Proposition 4.1, assume each $\left(X_{i}, v_{i}\right)$ is regular, and, for any $j \in J$, let $\mu_{j}$ be the nbd structure on $X$ defined by $\mu_{j}(x)=$ $f_{j}^{-1}\left(\nu_{j}\left(f_{j}(x)\right)\right)$. To show that $\mu_{j}$ is regular, let $A \in \mu_{j}(x)$. Then, there is $B \in \nu_{j}\left(f_{j}(x)\right)$ such that $f_{j}^{-1}(B) \subseteq A$. Since $v_{j}$ is regular, there is $C \in v_{j}\left(f_{j}(x)\right)$ such that $\mathrm{Cl}_{v_{j}} C \subseteq B$. By Proposition 2.9, $f_{j}\left(\mathrm{Cl}_{\mu_{j}}\left(f_{j}^{-1}(C)\right)\right) \subseteq \mathrm{Cl}_{\nu_{j}} f_{j}\left(f_{j}^{-1} C\right) \subseteq \mathrm{Cl}_{\nu_{j}} C$, and so $\mathrm{Cl}_{\mu_{j}} f_{j}^{-1}(C) \subseteq$ $f_{j}^{-1}\left(\mathrm{Cl}_{v_{j}} C\right) \subseteq A$. Since $f_{j}^{-1}(C) \in \mu_{j}(x),\left(X, \mu_{j}\right)$ is regular. As shown in the proof of Proposition 4.1, $\nu=\sup _{\mathrm{N}(X)}\left\{\mu_{\jmath}: J \in J\right\}$, and thus $(X, v)$ is regular by Proposition 3.2(2).

If each $\left(X_{j}, v_{j}\right)$ is supratopological, a simple argument shows that $\mu_{j}$ is a supratopology, and therefore $v$ is a supratopology by Proposition 3.2(1).

In the notation and terminology of Proposition 4.3, assume each $\left(X_{i}, v_{i}\right)$ is pretopological. To show that the final structure $\mu$ is pretopological, it suffices to show that $\mu(x)$ is a filter, for all $x \in X$. If $x \notin \bigcup_{i \in J} f_{i}\left(X_{i}\right), \mu(x)=\dot{x}$ is a filter. Let $x \in$ $\bigcup_{i \in J} f_{i}\left(X_{i}\right)$ and $A, B \in \mu(x)$. If $f_{i}(a)=x$, then $f^{-1}(A)$ and $f^{-1}(B)$ are in $v_{i}(a)$ and hence $f^{-1}(A \cap B) \in v_{i}(a)$. Thus $A \cap B \in \mu(x)$ and $\mu(x)$ is a filter.

Recalling the notations $\sigma v, \rho v$, and $\pi v$ for the supratopological, regular, and pretopological modifications of a given nbd structure $v$, we obtain the following corollary to Theorem 4.4 .

COROLlary 4.5. Let $f:(X, v) \rightarrow(Y, \mu)$ be continuous. Then so are the following:

(1) $f:(X, \sigma v) \rightarrow(Y, \sigma \mu)$;

(2) $f:(X, \rho v) \rightarrow(Y, \rho \mu)$;

(3) $f:(X, \pi v) \rightarrow(Y, \pi \mu)$.

Proof. (1) Since $\sigma \mu \leq \mu, f:(X, v) \rightarrow(X, \sigma v)$ is continuous, and so is $f:\left(X, v^{\prime}\right) \rightarrow$ $(X, \sigma \mu)$, where $v^{\prime}$ is the initial structure on $X$ induced by $(X, \sigma \mu)$ and $f$. But $\nu^{\prime}$ is a supratopology by Theorem 4.4, and since $\nu^{\prime} \leq \nu, v^{\prime} \leq \sigma v$.

(2) Similar to (1).

(3) Since $v \leq \pi v, f:(X, \pi v) \rightarrow(X, \mu)$ is continuous, and so is $f:(X, \pi v) \rightarrow\left(X, \mu^{\prime}\right)$, where $\mu^{\prime}$ is the final structure induced on $X$ by $(X, \pi \nu)$ and $f$. But $\mu^{\prime}$ is a pretopology by Theorem 4.4 , and since $\mu \leq \mu^{\prime}, \pi \mu \leq \mu^{\prime}$.

The next lemma is a rather obvious result; we omit the straightforward proof.

LEMMA 4.6. (1) Let $\left\{\left(X_{i}, v_{i}\right): i \in J\right\}$ be a collection of closure spaces and $\left\{f_{i}: i \in J\right\}$ a collection of maps $f_{i}: X_{i} \rightarrow X$. If $v$ is the final structure on $X$ in NBD induced by these collections, then $\sigma v$ is the final structure on $X$ in SUPTOP induced by the same collections.

(2) Let $\left\{\left(X_{i}, \mu_{i}\right): i \in J\right\}$ be a collection of pretopological spaces and $\left\{g_{i}: i \in J\right\} a$ collection of maps $g_{i}: X \rightarrow X_{i}$. If $\mu$ is the initial structure on $X$ in NBD induced by these collections, then $\pi \mu$ is the initial structure in PRTOP induced by these same collections.

THEOREM 4.7. Topological is an initial property in PRTOP and a final property in CLS.

Proof. Using the notation and assumptions of Lemma 4.6(2), assume that each $\left(X_{i}, \mu_{i}\right)$ is topological. By Theorem $4.4, \mu$ is a supratopology, and, by Lemma 4.6(2), $\pi \mu$ is the initial structure on $X$ relative to PRTOP which is, by Theorem 3.6(2), a topology. The second assertion is proved similarly, using Lemma 4.6(1) and Theorem 3.6(1). 
With the help of Theorem 4.4 and Corollary 4.5, we easily show that the modifications $\sigma, \rho$, and $\pi$ can be used to define functors mapping NBD onto CLS, RNBD, and PRTOP, respectively. The functors corresponding to $\sigma$ and $\rho$ are reflectors, the one corresponding to $\pi$ is a coreflector. However, when $\sigma$ is restricted to PRTOP, it follows by Theorem 4.7 that the functor determined by $\sigma$ is a reflector from PRTOP onto TOP, and, likewise, $\pi$ determines a coreflector from CLS onto TOP. These categorical results can be summarized as follows.

THEOREM 4.8. The categories NBD, PRTOP, CLS, TOP, and RNBD are topological. $C L S$ and RNBD are bireflective subcategories of NBD. PRTOP is a bicoreflective subcategory of NBD. TOP is a bireflective subcategory of PRTOP and a bicoreflective subcategory of CLS.

Subspaces and products are defined in the usual way as special cases of initial structures, coproducts and quotients as special cases for final structures. For future reference, we mention the following as consequences of Proposition 4.1.

Proposition 4.9. (1) If $(X, v)$ is an $n b d$ space and $A \subseteq X$, the subspace $\left(A, v_{A}\right)$ is described at each $x \in A$ by $v_{A}(x)=\cap\{A \cap V: V \in v(x)\}$.

(2) Let $\left\{\left(X_{i}, v_{i}\right): i \in J\right\}$ be a set of $n b d$ spaces and $X=\prod_{i \in J} X_{i}$ the Cartesian product set. The product nbd space $(X, v)=\Pi_{i \in J}\left(X_{i}, v_{i}\right)$ is characterized for each $x \in X$ by: $v(x)=\bigcup_{i \in J} P_{i}^{-1}\left(v_{i}\left(P_{i}(x)\right)\right)$ for all $x \in X$, where $P_{i}$ is the ith projection map.

By Theorem 4.4, Proposition 4.9 also describes subspaces and products of closure spaces. Alternate characterizations for these in terms of supraopen sets are given in the next proposition.

Proposition 4.10. (1) If $\left(A, v_{A}\right)$ is a subspace of the closure space $(X, v)$, then $\nu_{A}=\{V \cap A: V \in v\}$.

(2) If $(X, v)=\Pi_{i \in J}\left\{\left(X_{i}, v_{i}\right)\right\}$ where each $\left(X_{i}, v_{i}\right)$ is closure, then $v$ consists of all unions of sets of the form $P_{i}^{-1}\left(V_{i}\right), V_{i} \in v_{i}, i \in J$.

REMARK 4.11. The description of products in CLS contrasts with that in TOP, where sets of the form $P_{i}^{-1}\left(V_{i}\right), V_{i} \in v_{i}, i \in J$ constitute a subbase rather than a base for the product topology.

5. Some properties of neighborhood spaces. Various topological properties have been studied in the setting of pretopological spaces (e.g., see [2]), and more recently for closure spaces [5]. A more unified approach is to study these and other properties in the setting of nbd. spaces. Among the properties we consider is compactness, and it is interesting to note that the Tychonov theorem holds in NBD, whereas some other results familiar from topology do not.

Definition 5.1. Let $(X, v)$ be an nbd space, $A \subseteq X$. A collection $\mathscr{C}$ of subsets of $X$ is a $v$-neighborhood cover of $A$ if, for each $x \in A$, there is $V \in \mathcal{v}(x)$ such that $V \in \mathscr{C}$.

Definition 5.2. An nbd space $(X, v)$ is defined to be

(1) $T_{1}$ if $\cap v(x)=\{x\}$, for all $x \in X$;

(2) $T_{2}$ if distinct points have disjoint neighborhoods; 
(3) $T_{3}$ if regular and $T_{1}$;

(4) compact if each $v$-neighborhood cover of $X$ has a finite subcover;

(5) locally compact if each $v(x)$ contains a compact set (i.e., a set which is compact when considered as a subspace of $(X, v))$.

THEOREM 5.3. (1) $A T_{3}$ nbd space is $T_{2}$.

(2) An nbd space $(X, v)$ is $T_{2}$ if and only if each $v$-convergent $p$-stack has a unique limit.

Proof. (1) Assume $(X, v)$ is $T_{3}$ and $x \neq y$. Using $T_{1}$, choose $U \in v(x)$ such that $y \notin U$; then there is $V \in v(x)$ such that $\mathrm{Cl}_{v} V \subseteq U$, and $X \backslash V \in v(y)$ is disjoint from $V$.

(2) If $\mathscr{H} \stackrel{v}{\rightarrow} x, y$, then $v(x) \subseteq \mathscr{H}$ and $v(y) \in \mathscr{H}$ imply that $x$ and $y$ do not have disjoint neighborhoods. Conversely, if $(X, v)$ is not $T_{2}$, then there are $x, y$ in $X$ such that $V \in v(x)$ and $W \in v(y)$ imply that $W \cap V \neq \varnothing$. Thus $\mathscr{H}=v(x) \cup v(y)$ is a $p$-stack $v$-converging to both $x$ and $y$.

THEOREM 5.4. A subset $A$ of an $n b d$ space $(X, v)$ is compact if and only if each ultrafilter $F_{F}$ on $X$ containing $A$ is $v$-convergent to a point in $A$.

Proof. Let $(X, v)$ be compact, and assume there is an ultrafilter $\mathscr{F}$ containing $A$ which fails to converge to a point in $A$. Thus, for each $x \in X$, there is $V_{x} \in v(x)$ such that $V_{x} \notin \mathscr{F}$, and $U=\left\{V_{x}: x \in X\right\}$ is an nbd cover of $A$ with no finite subcover. Thus, $\mathscr{F}_{\text {F }}$ must converge to a point in $A$.

Conversely, if $U$ is a $v$-nbd cover of $A$ with no finite subcover, then $\mathscr{W}=\{A \backslash V$ : $V \in \mathscr{U}\}$ is a filter subbase, and if $\mathscr{G}$ is an ultrafilter containing [W], then $A \in \mathscr{G}$ but $\mathscr{G}$ cannot converge to any point in $A$.

THEOREM 5.5. The product $(X, v)=\Pi_{i \in J}\left(X_{i}, v_{i}\right)$ of a set of $n b d$ spaces is compact if and only if each component space $\left(X_{i}, v_{i}\right)$ is compact.

PRoof. We easily verify that a continuous image of a compact nbd space is compact, and hence each $\left(X_{i}, v_{i}\right)$ is compact if $(X, v)$ is compact. Conversely, assume each $\left(X_{i}, v_{i}\right)$ is compact and let $\mathscr{F}$ be an ultrafilter on $X$. Then $P_{i}(\mathscr{F})$ is an ultrafilter on $X_{i}$, which $v_{i}$-converges to some $x_{i} \in X_{i}$ by Theorem 5.4. If $x \in X$ is defined by $P_{i}(x)=x_{i}$, for all $i \in J$, then $\mathscr{F} \stackrel{v}{\rightarrow} x$ by Proposition 4 .2, and therefore, by Theorem $5.4,(X, v)$ is compact.

For closure spaces, compactness and regularity have more conventional characterizations. We omit these straightforward proofs.

THEOREM 5.6. Let $(X, v)$ be a closure space. Then

(1) $(X, v)$ is regular if and only if, whenever $A$ is supraclosed and $x \in X \backslash A$, there are disjoint supraopen sets $U, V$ such that $x \in U$ and $A \subseteq V$;

(2) $(X, v)$ is compact if and only if each cover of $X$ by supraopen sets has a finite subcover.

There are some well-known topological theorems involving separation properties and compactness which are not valid for nbd spaces. Easy examples can be found to demonstrate the following: 
(a) a closed subspace of a compact, $T_{2}$ nbd space need not be compact;

(b) a compact $T_{2}$ nbd space need not be regular;

(c) a product (in NBD or CLS) of two locally compact supratopological spaces need not be locally compact.

For (c), we can indeed choose each locally compact space to be the real line with its usual topology; the conclusion follows from the remark following Proposition 4.10.

Since ultra $p$-stacks play a role in nbd spaces analogous to that of ultrafilters in topological spaces, it is of some interest, in view of Theorem 5.4, to consider the following stronger version of compactness in which ultrafilters are replaced by ultra p-stacks.

Definition 5.7. A subset $A$ of an nbd space $(X, v)$ is $p$-compact if every ultrapstack containing $A v$-converges to a point in $A$. The space $(X, v)$ is $p$-compact if $X$ is a $p$-compact set.

Proposition 5.8. The continuous image of a $p$-compact set is $p$-compact.

Proof. Let $f:(X, v) \rightarrow(Y, \mu)$ be continuous, let $A \subseteq X$ be $p$-compact, and let $\mathscr{H}$ be an ultrapstack containing $f(A)$. If $\varphi$ is an ultrapstack containing the $p$-stack base $\left\{f^{-1}(H): H \in \mathscr{H}\right\} \cup\langle A\rangle$, then $\stackrel{\varphi}{\stackrel{v}{\rightarrow}} x$ for some $x \in A$, and $\mathscr{H}=f(\mathscr{G}) \stackrel{\mu}{\rightarrow} f(x) \in f(A)$, thus proving that $f(A)$ is $p$-compact.

THEOREM 5.9. A subset $A$ of an nbd space $(X, v)$ is $p$-compact if and only if each $\nu$-neighborhood cover of $A$ has a two-member subcover.

Proof. Suppose $\mathscr{H}$ is an ultrapstack containing $A$ which fails to $v$-converge to any point in $A$. Then for each $x \in A$, there is $U_{x} \in v(x)$ such that $U_{x} \notin \mathscr{H}$. By Lemma 2.2(c), $A \backslash U_{x} \in \mathcal{H}$, for all $x \in A$. Thus $U=\left\{U_{x}: x \in A\right\}$ is a $v$-neighborhood cover of $A$. But $U$ has no two-element subcover of $A$, for if $U, V \in \mathcal{U}$ and $A \subseteq U \cup V$, then $(A \backslash U) \cap(A \backslash V)=$ $A \backslash(U \cup V)=\varnothing$, contradicting the assumption that $\mathscr{H}$ is a $p$-stack.

Conversely, let $U$ be a $v$-neighborhood cover of $A$ with no two-element subcover of $A$. Then $\mathscr{B}=\{A \backslash U: U \in \mathcal{U}\}$ is $p$-stack base, and any ultrapstack containing $\mathscr{B}$ cannot converge to any point in $A$.

THEOREM 5.10. The product $(X, v)=\Pi_{i \in J}\left(X_{i}, v_{i}\right)$ of a set of $n b d$ space is $p$-compact if and only if each component space $\left(X_{i}, v_{i}\right)$ is $p$-compact.

Proof. Essentially the same as that of Theorem 5.5.

It is well known that Theorem 5.5 (the Tychonov theorem) holds for products in TOP and PRTOP, but this is not true for Theorem 5.10. Indeed, a two-element discrete topological space is $p$-compact and the product of this space with itself is $p$-compact relative to NBD and CLS, but not relative to TOP or PRTOP.

\section{REFERENCES}

[1] D. Andrijević, On b-open sets, Mat. Vesnik 48 (1996), no. 1-2, 59-64.

[2] G. Birkhoff, Lattice Theory, American Mathematical Society, New York, 1940.

[3] E. Čech, Topological Spaces, Interscience Publishers, New York, 1966. 
[4] G. Choquet, Convergences, Ann. Univ. Grenoble. Sect. Sci. Math. Phys. (N.S.) 23 (1948), 57-112.

[5] V. Claes, E. Lowen-Colebunders, and G. Sonck, Cartesian closed topological hull of the construct of closure spaces, Theory Appl. Categ. 8 (2001), no. 18, 481-489.

[6] D. Dikranjan, E. Giuli, and A. Tozzi, Topological categories and closure operators, Quaestiones Math. 11 (1988), no. 3, 323-337.

[7] F. Hausdorff, Grundzüge der Mengenlehre, Verlag von Veit \& Comp., Leipzig, 1914 (German).

[8] N. Levine, Semi-open sets and semi-continuity in topological spaces, Amer. Math. Monthly 70 (1963), 36-41.

[9] A. S. Mashhour, A. A. Allam, F. S. Mahmoud, and F. H. Khedr, On supratopological spaces, Indian J. Pure Appl. Math. 14 (1983), no. 4, 502-510.

[10] O. Njästad, On some classes of nearly open sets, Pacific J. Math. 15 (1965), no. 3, 961-970.

D. C. Kent: Department of Mathematics, Washington State University, Pullman, WA 99164-3113, USA

E-mail address: dkent@wsu .edu

Won Keun Min: Department of Mathematics, Kangwon National University, ChunCHEON, 200-701, KOREA

E-mail address: wkmin@cc. kangwon.ac. kr 


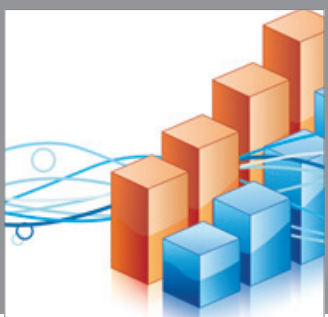

Advances in

Operations Research

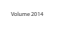

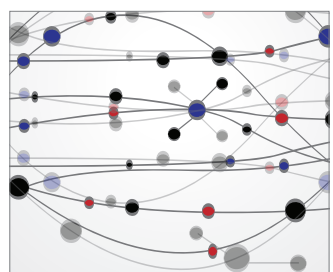

\section{The Scientific} World Journal
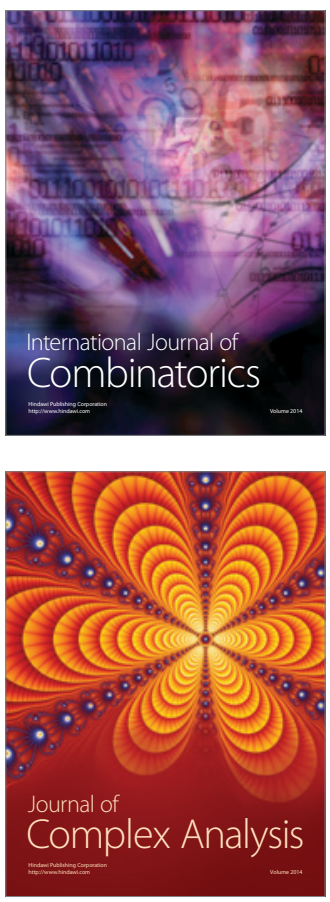

International Journal of

Mathematics and

Mathematical

Sciences
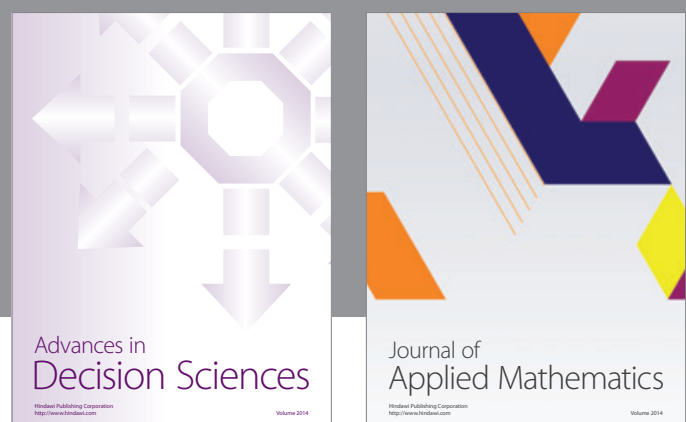

Journal of

Applied Mathematics
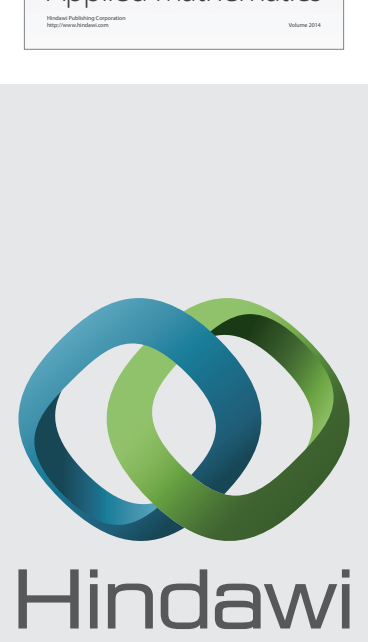

Submit your manuscripts at http://www.hindawi.com
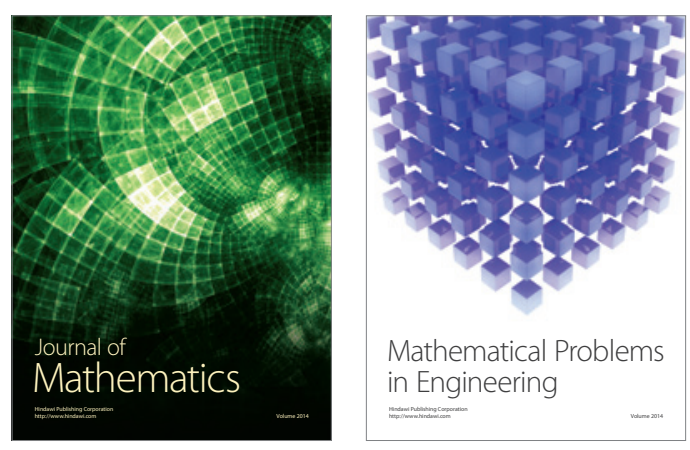

Mathematical Problems in Engineering
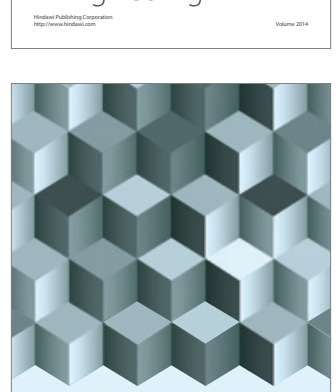

Journal of

Function Spaces
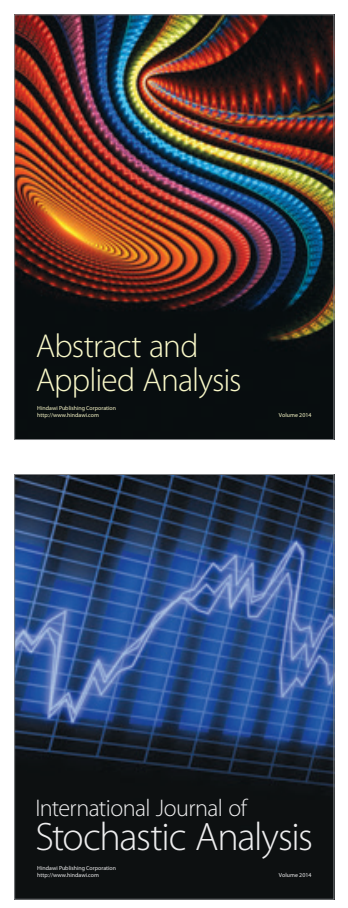

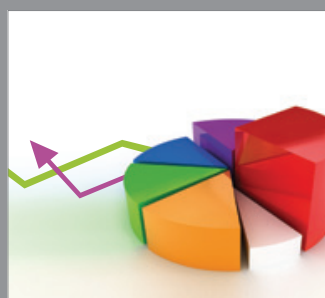

ournal of

Probability and Statistics

Promensencen
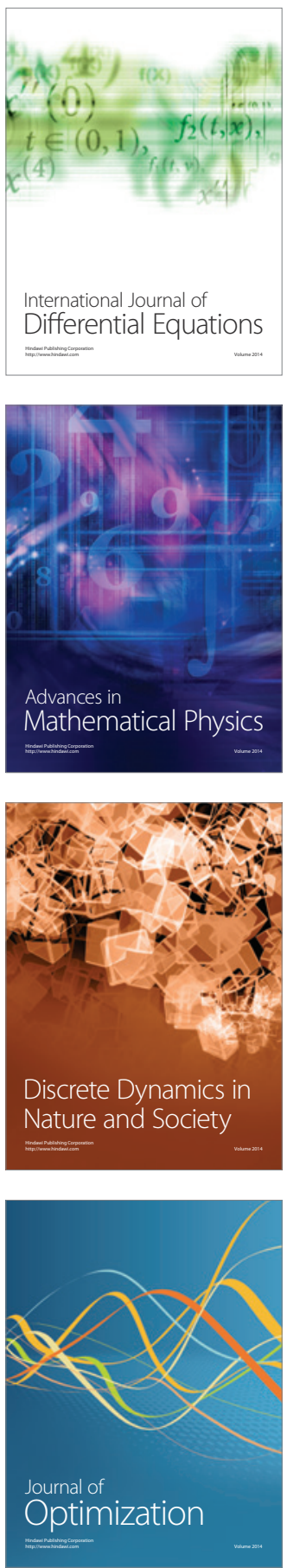